\title{
G. Giuliano, C. Criveller, M. Spivak, A. Frison (a cura di), Simvolizm i poetika pro- stranstva v tvorčestve Andreja Belogo, Nestor-Istorija, Sankt-Peterburg 2020, pp. 324.
}

Pellegrino e viaggiatore, Andrej Belyj ben si presta a letture e analisi che hanno nella spazialità e nella geografia il loro centro d'attenzione. Non soltanto per il suo contributo al testo pietroburghese con il romanzo Peterburg più volte analizzato (e mappato) dalla critica da un punto di vista spaziale, ma anche per la sua biografia, intrecciata strettamente alla pratica del viaggio. Oltre ai soggiorni in Europa, tra cui spiccano, per intensità e durata, quelli a Berlino e Dornach, sono celebri i viaggi non direttamente ascrivibili al cosiddetto Occidente. Belyj, infatti, partecipa attivamente alla costruzione di una peculiare declinazione 'meridionale' del concetto di Oriente, come nel caso della sua esperienza in Africa del Nord.

La slavistica italiana, che riveste un ruolo di primo piano nel campo degli studi belyjani a partire già dagli anni Settanta, con la pionieristica attività di pubblicazioni e convegni organizzati presso l'Università di Bergamo da Nina Kautschischwili, continua a produrre opere fondamentali nel campo. Tanto più che proprio il territorio italiano, e in particolare la Sicilia, aveva ospitato alcune tappe del viaggio-pellegrinaggio mistico intrapreso da Belyj nel ı9ı. A centosei anni di distanza da quell'itinerario e nel centenario della pubblicazione di Peterburg, nel dicembre 2016 è stato organizzato un particolarissimo 'convegno itinerante' tra Palermo e Monreale, con il sostegno delle Università di Padova, Salerno e Palermo, l'appoggio dell'Associazione Italiana degli Slavisti, il patrocinio della Regione Sicilia e della Casa-museo Andrej Belyj, filiale del Museo Statale A.S. Puškin di Mosca. Simvolizm i poetika prostranstva v tvorčestve Andreja Belogo, pubblicato da Nestor-Istorija e finanziato dal Dipartimento di Studi Linguistici e Letterari dell' Università degli Studi di Padova e dal Dipartimento di Studi Umanistici dell’Università di Salerno, raccoglie le ventitré relazioni presentate in quell'occasione.

Il volume è diviso in tre sezioni: nella prima sono analizzati gli scritti odeporici di Belyj, con un'attenzione particolare alla sua descrizione del territorio siciliano; la seconda, invece, si concentra su due luoghi geografici legati all'opera e alla biografia dello scrittore, le città di Mosca e Pietroburgo; nella terza, infine, lo sfondo spaziale è dato da ulteriori coordinate, sia tangibili e riconoscibili sulle mappe (il Caucaso, la provincia russa, l'Egitto), sia appartenenti a categorie critiche (le eterotopie, i nonluoghi, i luoghi inventati). Completa lo studio, a integrazione di quattro specifici articoli, una curata appendice grafica a colori che riporta testimonianze fotografiche del paesaggio urbano russo del primo Novecento, illustrazioni ai romanzi di Belyj e alcuni suoi disegni.

L'articolo che apre la prima sezione, a firma di N.A. Bogomolov, compianto studioso scomparso lo scorso novembre, può essere letto come introduzione all'intero volume, in quanto, ri- 
percorrendo gli itinerari di Belyj sullo sfondo delle pratiche di viaggio del periodo modernista, si interroga sul senso ultimo del suo vagabondare. All'analisi testuale di Putevye zametki sono dedicati i successivi due saggi di T. Nicolescu e G. Strano, che discutono rispettivamente il tema della composizione e delle revisioni dell'opera, analizzando meticolosamente il contenuto di tali mosaici letterari, e l'influsso steineriano sulla scrittura di Belyj, accompagnato da una puntuale trattazione della dimensione sonora e visuale dell'opera. Concentrati sullo specifico territorio siciliano sono invece i tre saggi successivi, scritti da D. Colombo, L. Szilárd e A. Visinoni. Nel primo l'autore, constatando l'assenza nel testo di Belyj dell'immagine della Sicilia a lui contemporanea, esamina in maniera comparata i cosiddetti orientalismi italiano e russo, nella fattispecie le ricadute letterarie delle narrazioni legate alla questione meridionale italiana e all'orientalizzazione interna russa. Szilárd concentra la sua attenzione sul luogo altamente simbolico di Monreale, polo opposto a Venezia, e analizza l'esperienza belyjana comparandola a quella, analoga, di Goethe. Visinoni approfondisce il peculiare spazio di Bagheria e il crogiolo di antichità greca, bizantina, cattolica e araba che vi si trova. Chiudono la prima sezione l'intervento di O.V. Marčenko, che mette in dialogo l'immagine dell' Italia di Belyj con quella del filosofo Vladimir Ern, e l'articolo di J. Ljuckanov, che indaga il Gesamtkunstwerk che emerge da varie opere odeporiche, in cui l'ekphrasis può essere intesa come forma di resistenza nei confronti di una narrazione marcatamente orientalista, pur senza uscire, tuttavia, da una visione prettamente eurocentrica.

La seconda sezione è costruita attorno a due luoghi urbani, Mosca e Pietroburgo, e la quasi totalità dei saggi è dedicata al romanzo Peterburg. L'intervento di O.M. Cooke esplora il tema egiziano (trasversale a tutta l'opera belyjana) nel romanzo, studiandone simboli (la Sfinge) e interpretazioni (antroposofica e mistica). O.A. Kling descrive i cambiamenti subiti dallo spazio geografico nelle varie redazioni del romanzo, considerando il ruolo del grottesco, sullo sfondo del legame intertestuale con le opere di Gogol' e Puškin. Le illustrazioni a Peterburg, sia quelle realizzate dall'autore del romanzo ma mai integralmente inserite nell'opera e conservate oggi nell'archivio del GLM (Gosudarstvennyj Literaturnyj Muzej), sia quelle effettivamente pubblicate a corredo del testo commissionate ad artisti e artiste già a partire dagli anni Venti, sono l'oggetto dell'indagine di E. Nasedkina. C. Criveller presenta le sue osservazioni preliminari attorno a un tema innovativo, il ruolo dello spazio nella sceneggiatura cinematografica del romanzo, incompiuta (e ancora inedita), composta dallo stesso Belyj, fornendo altresì informazioni sul rapporto tra lo scrittore e il mondo del cinema, e basando la propria interpretazione sulle coeve teorie formaliste. M. Ljunggren ripercorre le varie tradizionali letture critiche di Peterburg, definito "a changing chamaleon", a partire dal tema edipico. L'articolo di $\mathrm{O}$. Matich analizza il modo in cui la temporalità della narrazione del romanzo si incarna in forme spaziali, delineandone alcune modalità: l'uso dello sdvig, della metafora, e della metamorfosi barocca, prendendo anche in considerazione il saggio critico di Belyj Masterstvo Gogolja. Gli ultimi due interventi, non più strettamente concentrati su Peterburg, forniscono alla sezione un respiro più ampio: G. Giuliano esamina la dicotomia tra Mosca e Pietroburgo all' interno del testo Vtoraja simfonija e I. Delektorskaja teorizza un "arbatskij tekst" belyjano, descrivendone nel dettaglio le peculiarità spaziali e topografiche.

La terza sezione, la meno ancorata a schemi interpretativi tradizionali, si apre con il contributo di A. Cholikov che, nel solco di alcuni pionieristici lavori di D. Rizzi e T. Nicolescu composti nei primi anni Novanta, rende accessibili nuovi materiali relativi al mistero (incompiuto) Antichrist, ripercorrendone la genesi e analizzandone la dimensione spaziale. E. Gironi Carnevale presenta una lettura semiotica di un corpus di scritti belyjani neo-mitologici, pubblicati tra il I905 e il 1907 sulla rivista "Vesy", interpretati come esempi di eterotopie foucaltiane. L'articolo di R. 
Casari esamina il ruolo della città di Lichov del romanzo Serebrjanyj golub' in relazione allo spazio provinciale mercantile costruito in precedenza da Gogol' e Čechov. U. Persi, mutuando da Marc Augé il termine nonluoghi con opportuni aggiustamenti applica il concetto agli spazi (pre-natali, dell'io, del non-io) di Kotik Letaev. L'articolo di A. Frison, a partire dal concetto di Terzo Spazio di Bhabha, teorizza un peculiare e originale spazio stratigrafico all'interno dell'articolo Egipet composto da Belyj nel I912. Gli ultimi contributi si concentrano attorno allo scritto filosofico Istorija stanovlenija samosoznajušcej duši: $\mathrm{H}$. Stahl vi rintraccia una trattazione del mito di Parsifal, strettamente legato al paesaggio siciliano, e pubblica la terza parte dello scritto, corredata da puntuali note (l'intero trattato è attualmente in via di pubblicazione); M.P. Odesskij e M.L. Spivak ne analizzano l'elemento arabo e la sua funzione.

Le sovrapposizioni e le ripetizioni che emergono in alcuni punti del testo sono imperfezioni inevitabili quando si costruisce un progetto così composito, formato da tante e autorevoli voci differenti, tutte convergenti sulla stessa dimensione, quella spaziale. Il volume è un fondamentale contributo non soltanto agli studi su Belyj, ma anche al più generale tema del rapporto tra modernismo letterario e spazio geografico, e non è rivolto unicamente agli studiosi dello scrittore. Lo dimostra una recente video-recensione di Dmitrij Gasin, scrittore e videoblogger con più di 24000 iscritti al suo canale Youtube, vincitore del premio Revizor che, utilizzando l'hashtag naućpop, e sottolineando la dimensione plurale del progetto e il ruolo di primo piano dell'apporto scientifico italiano, presenta il volume proprio a un pubblico di non specialisti. 